\title{
Projections for HH measurements in the bbZZ(4I) final state with the CMS experiment at the HL-LHC
}

\section{Elisa Fontanesi*, Lisa Borgonovi}

University of Bologna and INFN

E-mail: elisa.fontanesi@cern.ch, lisa.borgonovi@cern.ch

\begin{abstract}
Prospects for the study of Higgs boson pair $(\mathrm{HH})$ production in the $\mathrm{HH} \rightarrow \mathrm{b} \bar{b} 4 \mathrm{l}(\mathrm{l}=\mathrm{e}, \mu)$ channel are studied in the context of the High Luminosity LHC. The analysis is performed using a parametric simulation of the Phase-2 CMS detector response provided by the Delphes software and assuming an average of 200 proton-proton collisions per bunch crossing at a center-of-mass energy of $14 \mathrm{TeV}$. Assuming a projected integrated luminosity of $3000 \mathrm{fb}^{-1}$, the expected significance for the nonresonant standard model (SM) HH signal is $0.37 \sigma$; a 95\% confidence level (CL) upper limit on its cross section is set to 6.6 times the SM prediction. The statistical combination of five decay channels ( $b \bar{b} b \bar{b}, b \bar{b} \tau \tau, b \bar{b} \gamma \gamma, b \bar{b} W W, b \bar{b} Z Z$ ) results in an expected significance for the SM HH signal of $2.6 \sigma$ and an expected $68 \%$ and 95\% CL intervals for the self-coupling modifier $\kappa_{\lambda}=\lambda_{H H H} / \lambda_{H H H}^{S M}$ of $[0.35,1.9]$ and $[-0.18,3.6]$, respectively.
\end{abstract}

7th Annual Conference on Large Hadron Collider Physics - LHCP2019

20-25 May, 2019

Puebla, Mexico

${ }^{*}$ Speaker. 


\section{Introduction}

The CERN LHC future physics program will target a large range of measurements, including a detailed study of the Higgs boson properties and direct searches for physics beyond the standard model (BSM). The measurement of the Higgs pair production is an important test of the standard model (SM) electroweak symmetry breaking (EWSB) sector, because it allows to extract the Higgs self-coupling $\left(\lambda_{H H H}\right)$, which is directly related to the structure of the Higgs field potential. Furthermore, any possible deviations in the Higgs self-coupling due to BSM effects could open the door to new physics searches and provide important tests of the validity of the SM. Hence, a parametrization of an anomalous coupling $\lambda_{H H H}=\kappa_{\lambda} \cdot \lambda_{H H H}^{S M}$ has been introduced, where $k_{\lambda}$ is called self-coupling modifier.

In hadron colliders such as LHC, the dominant nonresonant HH production mode proceeds through top-quarks loop diagrams in the gluon fusion channel, shown in Figure 1. Their contribution is affected by a destructive interference between the box and the triangle diagrams; consequently, the total SM production rate of the double Higgs process is really small (31.05 fb and $36.69 \mathrm{fb}$ at a center-of-mass energy of $13 \mathrm{TeV}$ and $14 \mathrm{TeV}$, respectively) [1].
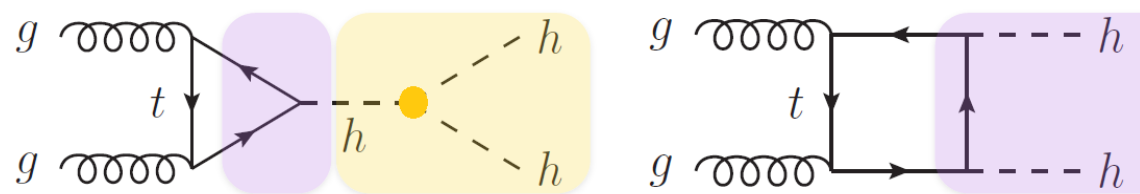

Figure 1: Leading order Feynman diagrams for nonresonant HH production in the SM through the Higgs boson self-coupling (left) and the top-box diagram (right).

Here, a study of the $\mathrm{HH} \rightarrow \mathrm{b} \bar{b} Z Z^{*}(41)$ channel in proton-proton collisions at the HL-LHC at a center-of-mass energy of $14 \mathrm{TeV}$ is presented considering the Phase-2 CMS detector. The upgrade programme of the CMS detector, necessary to fully exploit the physics potential of the LHC, has been designed to cope with an istantaneous luminosity up to $\mathscr{L}=5 \times 10^{34} \mathrm{~cm}^{-2} \mathrm{~s}^{-1}$ and an average number of pileup events up to 200 .

In the final section, results obtained from the statistical combination of five decay channels $(\mathrm{b} \bar{b} b \bar{b}$, $\mathrm{b} \overline{\mathrm{b}} \tau \tau, \mathrm{b} \overline{\mathrm{b}} \gamma \gamma, \mathrm{b} \overline{\mathrm{b}} \mathrm{WW}, \mathrm{b} \overline{\mathrm{b} Z Z}$ ) are presented; all these analyses are designed to be orthogonal.

\section{The $H H \rightarrow b \bar{b} Z Z^{*}(4 \mathbf{l})$ analysis at the HL-LHC}

Up to now, the low signal rate of $\mathrm{HH}$ events leads to consider mostly final states with a sizable branching ratio. In view of HL-LHC, some rare but clean processes have been re-considered because of the increasing available statistics and the challenging conditions due to the enormous number of pileup events. In this work, the sensitivity to the Higgs self-coupling for $\mathrm{m}_{H}=125 \mathrm{GeV}$ is

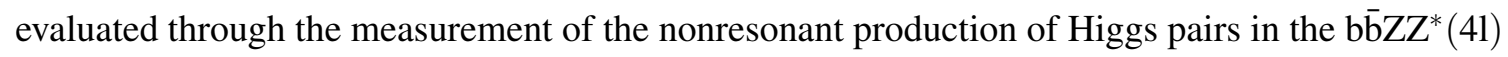
final state. Despite a small cross section $\left(\sigma_{\mathrm{b} \bar{b} 41}=5.3 \mathrm{ab}\right)$, the presence of four leptons associated with two $b$ jets leads to a very clean final state topology allowing to maintain a rather good signal selection efficiency and to control the backgrounds. 


\section{Monte Carlo samples}

Signal events are generated at leading order (LO) with MadGraph5_aMC@NLO [2] accounting for the full $m_{t}$ dependence, for several values of $k_{\lambda}$ ranging from $k_{\lambda}=-10$ to $k_{\lambda}=10$, in steps of 0.5. The single Higgs boson production in gluon ( $\mathrm{ggH}$ ) and vector boson (VBF) fusion, and in associated production with top quarks ( $\mathrm{t} \overline{\mathrm{t}} H$ ) and vector bosons $(\mathrm{VH})$, is considered as a background for $\mathrm{HH}$ production. The main contribution to the background comes from $\mathrm{t} \overline{\mathrm{t}}(\mathrm{b} \overline{\mathrm{b}}) \mathrm{H}(41), \mathrm{t} \bar{Z}(2 \mathrm{l})$, $\operatorname{ggH}(41)$ events, followed by minor contributions from $\mathrm{Z}(\mathrm{b} \bar{b}) \mathrm{H}(41), \mathrm{WH}(4 \mathrm{l})$ and $\mathrm{VBF}(\mathrm{H})$; $\operatorname{tt} Z Z(41)$ is found to be negligible. $\operatorname{ggH}$ and $\operatorname{VBF}(\mathrm{H})$ lead to a significant contribution to the final state b̄ ZZZ(4l) mainly because of the large number of pileup events. The background processes are generated at LO with MadGraph5_aMC@NLO, except ggH and VBF(H), generated with POWHEG [3]. All simulated samples are normalised to the expected SM cross section as recommended in [4]: samples production cross sections are summarized in Table 1. Generated signal and background samples are showered and hadronized with Pythia8 [5] and processed with the Delphes fast simulation software [6], used to model the Phase-2 CMS detector and simulate an average number of pileup events of 200 .

\begin{tabular}{c|c|c|c|c|c|c}
$\mathrm{HH}$ & $\mathrm{t} \overline{\mathrm{t}} \mathrm{H}$ & $\mathrm{t} \overline{\mathrm{t} Z}$ & $\mathrm{ZH}$ & $\mathrm{WH}$ & $\mathrm{VBF}(\mathrm{H})$ & $\mathrm{ggH}$ \\
\hline 0.0053 & 0.0761 & 69.224 & 0.0183 & 0.1876 & 1.1690 & 15.007
\end{tabular}

Table 1: Cross section times branching ratio [fb] for the signal and the background processes.

\section{Event selection}

At least four identified and isolated muons (electrons) with $|\eta|<2.8$ and $p_{T}>5$ (7) $\mathrm{GeV}$ are required in the events, where muons and electrons are selected if passing loose (medium) identification criteria with a relative isolation smaller than 0.7. $Z$ boson candidates are formed from pairs of opposite-charge leptons $\left(l^{+} l^{-}\right)$requiring a minimum angular separation between two leptons of 0.02. At least two dilepton pairs are required. The $Z$ candidate with the invariant mass closest to the nominal $Z$ mass is denoted as $Z_{1}$; then, among the other opposite-sign lepton pairs, the one with the highest $p_{T}$ is labelled as $Z_{2}$. In order to improve the sensitivity to the Higgs boson decay, $Z$ candidates are required to have an invariant mass in the range $[50,100] \mathrm{GeV}\left(Z_{1}\right)$ and $[12$, 60] $\mathrm{GeV}\left(Z_{2}\right)$, respectively. At least one lepton is required to have $p_{T}>20 \mathrm{GeV}$ and a second is required to have $p_{T}>10 \mathrm{GeV}$. The four leptons invariant mass, $m_{4 l}$, is requested to be in the range $120<m_{4 l}<130 \mathrm{GeV}$. Two or three identified $\mathrm{b}$ jets, reconstructed with the anti- $k_{T}$ algorithm inside a cone of radius $R=0.4$, are required; a b-tag medium working point, exploiting the presence of the MIP Timing Detector (MTD), is assumed. Their invariant mass is required to be in the range $90<m_{b \bar{b}}<150 \mathrm{GeV}$ and the angular distance between the two $\mathrm{b}$ jets has to be $0.5<\Delta R_{b \bar{b}}<2.3$; furthermore, a missing transverse energy (MET) cut is fixed at $150 \mathrm{GeV}$ and $\Delta R_{H H}>2.0$.

\section{Results}

The invariant mass spectrum of the four leptons after the full event selection is shown in Figure 2a. The expected event yields, shown in Table 2, are normalised to an integrated luminosity of $3000 \mathrm{fb}^{-1}$ for the HH signal and the considered background processes. The most sensitive channel 
is $\mathrm{b} \overline{\mathrm{b}} 4 \mu$, but a sizeable contribution to the sensitivity also comes from the $\mathrm{b} \overline{\mathrm{b}} 2 \mathrm{e} 2 \mu$ and $\mathrm{b} \overline{\mathrm{b}} 4 \mathrm{e}$ final states. The main sources of systematic uncertainty are related to the muon/electron reconstruction, identification and isolation ( $0.5 \%$ in both cases), to the $\mathrm{b}$ tagging algorithm (ranging from $1 \%$ to $6 \%$ ) and to the integrated luminosity (1\%). The impact of the systematic uncertainties on the analysis is found to be almost negligible. Including all the considered final states, the combined upper limit at the $95 \% \mathrm{CL}$ on the $\mathrm{HH}$ cross section corresponds to 6.6 times the SM prediction, with a corresponding significance of $0.37 \sigma$. A scan of the negative log-likelihood as a function of the signal strength $\mu=\sigma_{H H} / \sigma_{H H}^{S M}$ in the $b \overline{b Z Z} Z^{*}(41)$ channel is shown in Figure $2 \mathrm{~b}$ (orange line), where the presence of a signal with the strength and properties predicted by the SM is assumed.

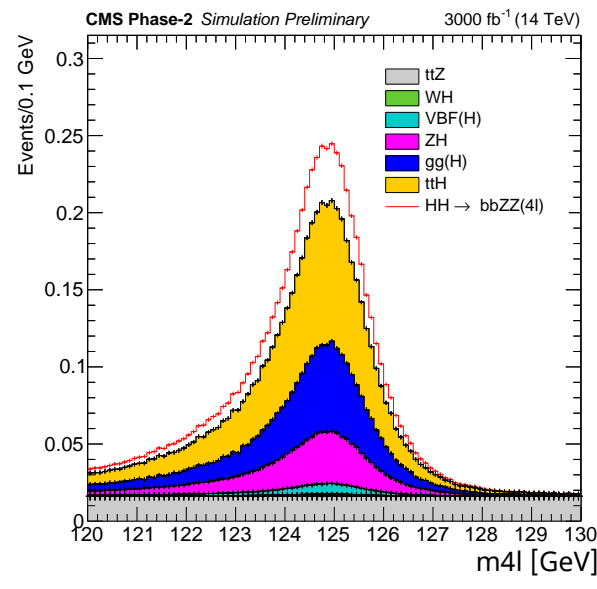

(a)

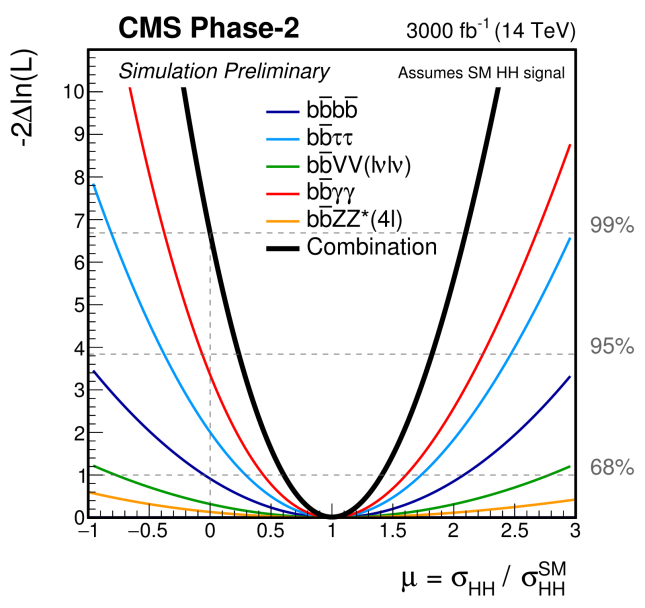

(b)

Figure 2: Invariant mass distribution of the four leptons selected at the end of the analysis for the signal (in red) and the considered background processes (a); scan of the negative log-likelihood as a function of the signal strength $\mu$ assuming SM HH signal in the five decay channels analysed and their combination (b).

\begin{tabular}{l|ccccccc} 
& $\mathrm{HH}$ & $\mathrm{t} \overline{\mathrm{t}} \mathrm{H}$ & $\mathrm{ggH}$ & $\mathrm{ZH}$ & $\mathrm{WH}$ & $\mathrm{VBF}$ & $\mathrm{t} \overline{\mathrm{Z}}$ \\
\hline$b \bar{b} 4 l$ & 1.0 & 2.5 & 1.5 & $9.4 \cdot 10^{-1}$ & $4.0 \cdot 10^{-2}$ & $1.7 \cdot 10^{-1}$ & 1.6 \\
$b \bar{b} 4 \mu$ & $4.9 \cdot 10^{-1}$ & 1.3 & $6.9 \cdot 10^{-1}$ & $4.9 \cdot 10^{-1}$ & $2.2 \cdot 10^{-2}$ & $1.1 \cdot 10^{-1}$ & $8.1 \cdot 10^{-1}$ \\
$b \bar{b} 4 e$ & $8.8 \cdot 10^{-2}$ & $2.2 \cdot 10^{-1}$ & $5.3 \cdot 10^{-2}$ & $6.9 \cdot 10^{-2}$ & $2.9 \cdot 10^{-3}$ & $1.1 \cdot 10^{-2}$ & 0.0 \\
$b \bar{b} 2 e 2 \mu$ & $4.2 \cdot 10^{-1}$ & 1.0 & $7.6 \cdot 10^{-1}$ & $3.8 \cdot 10^{-1}$ & $1.5 \cdot 10^{-2}$ & $4.9 \cdot 10^{-2}$ & $7.9 \cdot 10^{-1}$
\end{tabular}

Table 2: Event yields for the signal and the background processes, normalised to $3000 \mathrm{fb}^{-1}$.

\section{Combination results}

The statistical combination of five decay channels (b $\bar{b} b \bar{b}, b \bar{b} \tau \tau, b \bar{b} \gamma \gamma, b \bar{b} W W, b \bar{b} Z Z)$ results in a combined $95 \%$ CL upper limit on the SM HH cross section of 0.77 times the SM prediction. The absence of a HH signal, corresponding to $\mu=0$, is excluded at the 99\% (2.6 $\sigma$ ) CL. Both systematic and statistical uncertainties are considered. Results for each considered final state are shown in Table 3 and details of each analysis are documented in [7].

Prospects are also studied for the measurement of the trilinear Higgs boson coupling for each final state and their combination (Figure 3a). The expected $68 \%$ and $95 \%$ confidence level inter- 


\begin{tabular}{|c|c|c|c|c|c|c|}
\hline $\mathrm{HH} \rightarrow$ & bb̄bb & $\mathrm{b} \overline{\mathrm{b}} \tau \tau$ & $\mathrm{b} \overline{\mathrm{b}} \gamma \gamma$ & $\mathrm{b} \bar{b} \mathrm{VV}(1 v l v)$ & $b \bar{b} Z Z^{*}(41)$ & Combination \\
\hline Significance $(\sigma)$ & 0.95 & 1.4 & 1.8 & 0.56 & 0.37 & 2.6 \\
\hline Limit at $95 \%$ CL & 2.1 & 1.4 & 1.1 & 3.5 & 6.6 & 0.77 \\
\hline
\end{tabular}

Table 3: Significance and 95\% CL upper limit for each channel analysed and their combination (in black).

vals for the self-coupling modifier $\kappa_{\lambda}=\lambda_{H H H} / \lambda_{H H H}^{S M}$ are $[0.35,1.9]$ and $[-0.18,3.6]$, respectively. Under the assumption that no HH signal exists, 95\% CL upper limits on the SM HH production cross section are derived as a function of $\kappa_{\lambda}$ (Figure $3 b$ ): the excluded cross section changes as a function of $\lambda_{H H H}$, because it is directly related to variations in the HH kinematic properties.

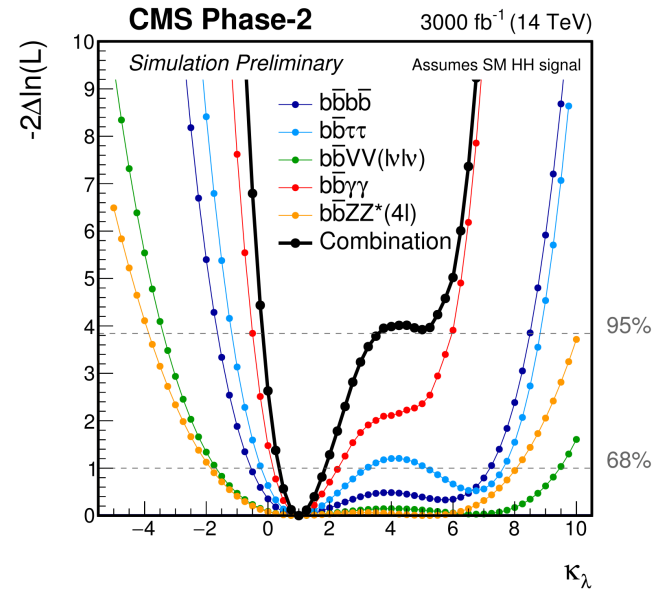

(a)

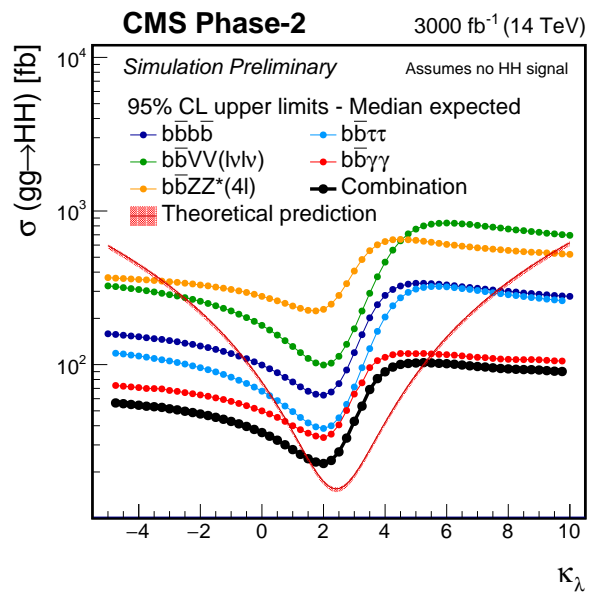

(b)

Figure 3: Expected likelihood scan (a) and upper limit at the 95\% CL on the HH production cross section (b) as a function of $\kappa_{\lambda}$ assuming SM HH signal and absence of HH signal, respectively. The functions are shown separately for the five decay channels studied and for their combination (in black).

\section{References}

[1] A. Azatov. R. Contino, G. Panico, M. Sonb, Effective field theory analysis of double Higgs production via gluon fusion, CERN-PH-TH-2015-015.

[2] J. Alwall at al., The automated computation of tree-level and next-to-leading order differential cross sections, and their matching to parton shower simulations, arXiv:1405.0301.

[3] S. Frixione, P. Nason, and G. Ridolfi, A Positive-weight next-to-leading-order MonteCarlo for heavy flavour hadroproduction, JHEP09(2007) 126, doi:10.1088/1126-6708/2007/09/126, arXiv:0707.3088.

[4] D. de Florian et al., Handbook of LHC Higgs cross sections: 4. Deciphering the nature ofthe Higgs sector, CERN Report CERN-2017-002-M, 2016.doi:10.23731/CYRM-2017-002, arXiv:1610.07922.

[5] T. Sjöstrand et al., An introduction to PYTHIA 8.2, Computer Physics Communications 191 (2015) 159, doi:10.1016/j.cpc.2015.01.024.

[6] J. de Favereau et al., DELPHES 3, A modular framework for fast simulation of a generic collider experiment, JHEP 1402 (2014) 057.

[7] The CMS Collaboration, CMS-PAS-FTR-18-019. 